Jona Minette E. Ligon, MD

Natividad A. Almazan, MD, MSc

Department of Otorhinolaryngology

Head and Neck Surgery

East Avenue Medical Center
Correspondence: Dr. Jona Minette E. Ligon

Department of Otorhinolaryngology

Head and Neck Surgery

6th Floor, East Avenue Medical Cente

East Avenue, Diliman, Quezon City 1100

Philippines

Phone: (632) 9280611 local 324

Fax: (632) 4356988

Email: ladyarcher11@yahoo.com

The authors declared that this represents original material that is not being considered for publication or has not been published or accepted for publication elsewhere in full or in part, in print or electronic media; that the manuscript has been read and approved by the authors, that the requirements for authorship have been met by the authors, and that the authors believe that the manuscript represents honest work.

Disclosures: The authors signed disclosures that there are no financial or other (including personal) relationships, intellectual passion, political or religious beliefs, and institutional affiliations that might lead to a conflict of interest.

Presented at the Philippine Society of Otolaryngology Head and Neck Surgery Analytical Research Contest. October 23, 2014 Unilab Bayanihan Center, Pasig City.

\section{The Effectiveness of Intravenous Tranexamic Acid on Blood Loss and Surgical Time During Endoscopic Sinus Surgery: A Systematic Review}

\begin{abstract}
Objective: To review the effectiveness of intravenous tranexamic acid in reduction of blood loss, surgical time and field visualization among patients who underwent endoscopic sinus surgery (ESS) for chronic rhinosinusitis (CRS).
\end{abstract}

\section{Methods:}

Data Sources: MEDLINE (PubMed), EMBASE, ScienceDirect, HERDIN, and the Cochrane Library.

Eligibility Criteria: Randomized controlled trials (RCT) between 2005-2014 that evaluated the effects of tranexamic acid or placebo in patients undergoing ESS for CRS.

Appraisal and Synthesis Methods: Articles were selected by 2 independent reviewers and methodological quality was blindly evaluated using a Jadad scale. Data were compiled in tables for analysis of outcome measures (estimated blood loss, length of surgery and intraoperative surgical field visualization).

Results: Two trials were included in the study, enrolling 128 patients. One arm of the study had been given tranexamic acid while the other arm was given placebo (saline solution). Results varied for both studies. The summary of the observed difference for blood loss had a standardized mean difference of $-51.20\left(\mathrm{Cl}_{95}[-59.44,-42.95]\right)$ showing that the blood loss in milliliters was less in the tranexamic group compared to saline solution. The summary of the observed difference in surgical time had a standardized mean difference of $-19.32\left(\mathrm{Cl}_{95}[-24.21,-14.43]\right)$ showing that the surgical time in minutes was shorter in the tranexamic group compared to saline solution. The secondary outcome on surgical field visualization was not pooled together because the studies used different measurement scales.

Limitations: The most important weaknesses of the 2 included studies were the differences in dose of tranexamic acid, scales of measurement of field visibility and age groups of the patients.

Conclusion: Tranexamic acid reduced blood loss and shortened surgical time after ESS among patients with CRS. However, the additional benefit of tranexamic acid for better field visualization was not clear. Adverse effects were not considered in this study, however, results support the use of intravenous tranexamic acid intraoperatively as an option for ESS with blood loss as a concern. Further randomized clinical trials and an update on the systematic review will strengthen the evidence on the effectivity of tranexamic acid for ESS. 


\section{Keywords: tranexamic acid, endoscopic sinus surgery, bleeding time}

Endoscopic Sinus Surgery (ESS) is a useful and widespread technique, popular among otolaryngologists, that allows the treatment of a large number of nasal pathologies with the aim of maintaining physiological function and anatomical structure. ${ }^{1}$ The most common complication during ESS is bleeding ${ }^{2}$ which in excess, can hinder completion of surgery by reducing intraoperative visualization of the field. Various techniques (including hypotensive anesthesia, reverse Trendelenberg positioning, and administration of local vasoconstrictors) have been used by surgeons to reduce this complication. 1,3,4

Tranexamic acid is an antifibrinolytic drug that can be administered to decrease intraoperative bleeding. In the clotting cascade, it serves to stabilize the fibrin clot to reduce bleeding. ${ }^{5}$ Tranexamic acid has been used extensively in certain surgical procedures and has been shown to limit bleeding with no increase in adverse events. ${ }^{5}$

There have been many studies on the effect of tranexamic acid in reducing blood loss in surgeries ${ }^{6-8}$ but we were particularly interested in those involving ESS. The aim of this study is to determine the effectiveness of intraoperative intravenous tranexamic acid in comparison to normal saline in reduction of blood loss and duration of operation among patients undergoing endoscopic sinus surgery. Surgical field visualization was also compared as a secondary outcome.

\section{METHODS}

\section{Protocol and Registration}

The methods for analysis and criteria for this review were specified and documented in advance. This secondary research protocol was neither registered nor reviewed by an Institutional Review Board.

\section{Eligibility Criteria}

Studies: Randomized clinical trials (RCT) in English on the effects of intravenous tranexamic acid compared to placebo in sinus surgeries from 2005 to 2014 were considered for inclusion.

Participants: Patients of both sexes and all ages who underwent ESS for CRS were included. Those who underwent previous ESS, had cardiovascular pathology, bleeding disorders, hypertension, kidney or liver dysfunction, anemia or were taking anti-coagulants were excluded.

Interventions: Trials comparing the effect of intraoperative intravenous tranexamic acid and saline solution (placebo) were included. The trials given oral or topical tranexamic acid were excluded.

Outcome Measures: The primary outcomes were intraoperative blood loss and surgical time. The secondary outcome was visualization of the surgical field. The Wormald ${ }^{9}$ grading scale used by Langille et al. ${ }^{5}$ and the 5-point scale adapted from Boezaart et al. ${ }^{10}$ by Eldaba et al. ${ }^{2}$ were used in this study.

\section{Information Sources and Search Methods}

We searched the following electronic bibliographic databases MEDLINE (PubMed), EMBASE, ScienceDirect, HERDIN and the Cochrane Library (Cochrane Database of Systematic Reviews, CDSR). We included studies in English published between 2005 and 2014. Several databases were used to ensure that relevant articles were identified, as publication bias was more likely to be found if only one to two databases were used. The following subject headings and text words, and their combinations, were included in the MEDLINE search strategy: "bleeding AND endoscopic sinus surgery", "tranexamic acid AND randomized clinical trials," "bleeding AND functional endoscopic sinus surgery", "bleeding AND endoscopic sinus surgery AND randomized clinical trials", "tranexamic acid OR functional endoscopic sinus surgery AND randomized clinical trials", "tranexamic acid AND randomized control trials AND sinus surgery", "bleeding OR functional endoscopic sinus surgery AND tranexamic acid AND randomized clinical trials". The files and library of East Avenue Medical Center Department of Otolaryngology-Head and Neck Surgery and main libraries were also searched for unpublished papers with the same topic. Additionally, we searched manually through the reference lists of the identified articles.

\section{Study Selection}

The search included all randomized controlled trials comparing intraoperative intravenous tranexamic acid and placebo (saline solution) among patients who underwent ESS. There were no restrictions on eligibility according to drug dosing, duration given and we did not exclude specific populations or age groups.

\section{Screening process}

Two independent reviewers screened the citation titles and abstracts using a predesigned form. Titles and abstracts that clearly did not meet inclusion criteria were excluded. For titles fulfilling inclusion criteria, full-text articles were obtained. Conflicting results were resolved by consulting a third reviewer.

\section{Assessment of Methodological Quality}

Studies that met the criteria for inclusion were assessed independently by 2 reviewers for methodological quality using the Jadad scale. ${ }^{11,12}$ Randomization, blinding and dropouts were verified in each study. The range of possible scores was 0 (bad) to 5 (good). Studies with a score of 4 or higher in the Jadad scale were included in the study.

\section{Data Abstraction and Analysis}

The data was abstracted in duplicate. Information was extracted from the original reports on standardized forms. All data was entered in Review Manager 5 (RevMan v.5.2, The Cochrane Collaboration, 2012, Copenhagen). The primary outcomes were amount of blood loss and surgical time. The secondary outcome was visualization of the field. There was no access to individual data from the authors so we used the summary data provided in the publication. 
The Jadad scale to assess risk of bias was independently used by the 2 reviewers. There were no disagreements. The fixed effect model was used to combine the treatment effect estimates from the individual studies. The combined estimate of treatment effect was reported with $95 \%$ confidence interval. Between-study heterogeneity was assessed using $\mathrm{I}^{2}$ measure and a formal hypothesis test. Forest plots showing the effect estimates of the individual studies and the combined effect allowed visual assessment of heterogeneity. Subgroup analysis was not carried out for the different outcomes.

The standardized difference in means of blood loss and duration of operation comparing intravenous tranexamic acid to placebo (saline solution) was the primary measure of treatment effect. For the secondary outcome of visualization of surgical field, the 2 studies assessed the same outcome but was measured using 2 different score scales (Wormald ${ }^{9}$ and Boezaart ${ }^{10}$ Score scales) so the results were not pooled but reported individually.

\section{RESULTS}

\section{Search Results and Study Selection}

Overall 8 potentially relevant publications were initially identified. Amongst these, 6 trials were excluded because of different comparators and outcome measures. Therefore, 2 trials were finally evaluated. (Figure 1)

\section{Study characteristics and Assessment of Reporting}

The characteristics of the 2 included studies are summarized in Table 1.

The 2 relevant studies enrolled a total of 128 patients, 100 children aged 5-10 years old and 50 adults aged 28 to 78 years old. Results of these studies are summarized in Table 2.

The study of Langille, et al. was a double blind, randomized, placebo controlled trial and had a population of 28 adults aged 23-80 years old, with a median age of 45 enrolled between March 2010 and November 2011. Block randomization was used and only 1 study investigator knew the randomization and was responsible for preparing the tranexamic acid or saline solutions. The said investigator was not involved in data extraction or analysis. The surgeon and anesthesiologists were also blinded to the treatment given. Half of the participants (14) were given a tranexamic acid bolus of $15 \mathrm{mg} / \mathrm{kg}$ then infusion of $1 \mathrm{mg} / \mathrm{kg} /$ $\mathrm{hr}$, while the other half were given normal saline. The study showed no statistically significant difference between groups in estimated blood loss ( $p=.40)$, surgical time $(p=.14)$ and surgical field visualization $(p=.89) .{ }^{5}$ The Wormald grading scale ${ }^{9}$ was used to measure the surgical field visualization with the mean of 5.84 vs 5.8 for the tranexamic acid and normal saline groups, respectively.

The study of Eldaba, et al. had 100 children aged 5-10 years, who

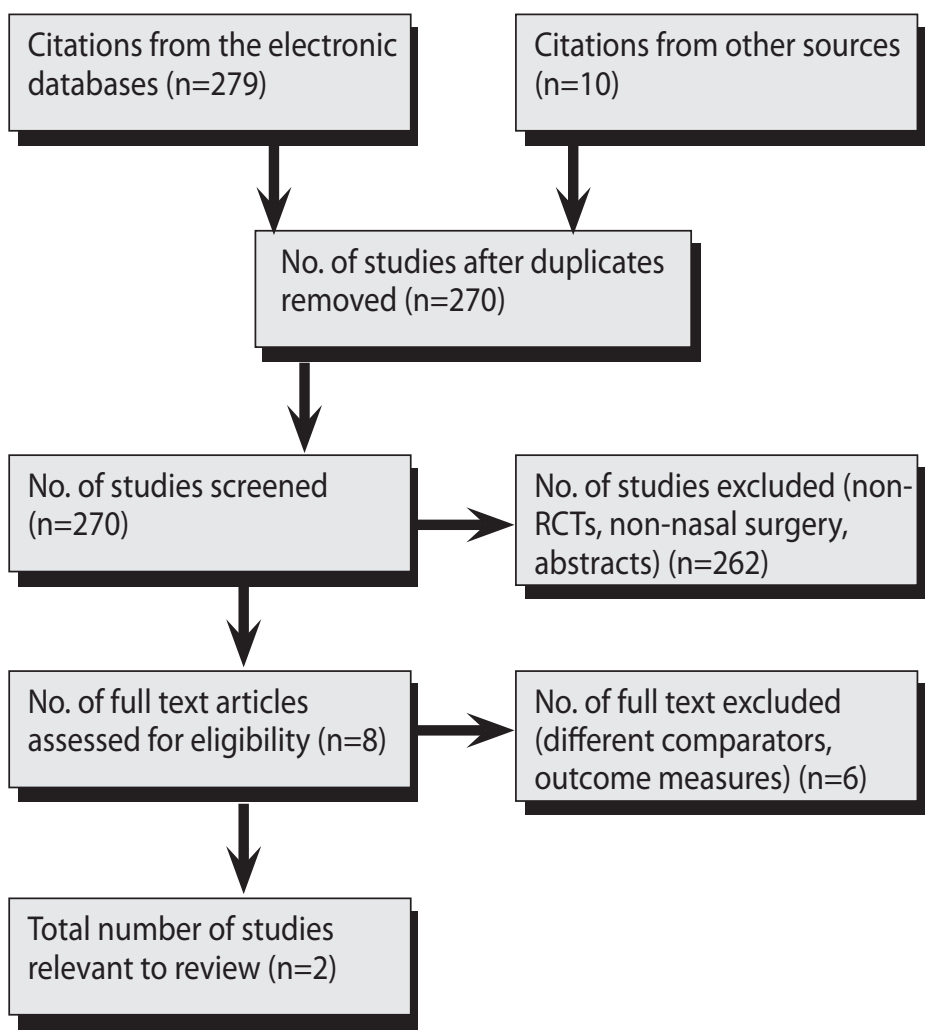

Figure 1. Flow Chart for selection of randomized controlled trials

Table 1. Characteristics of Included Studies

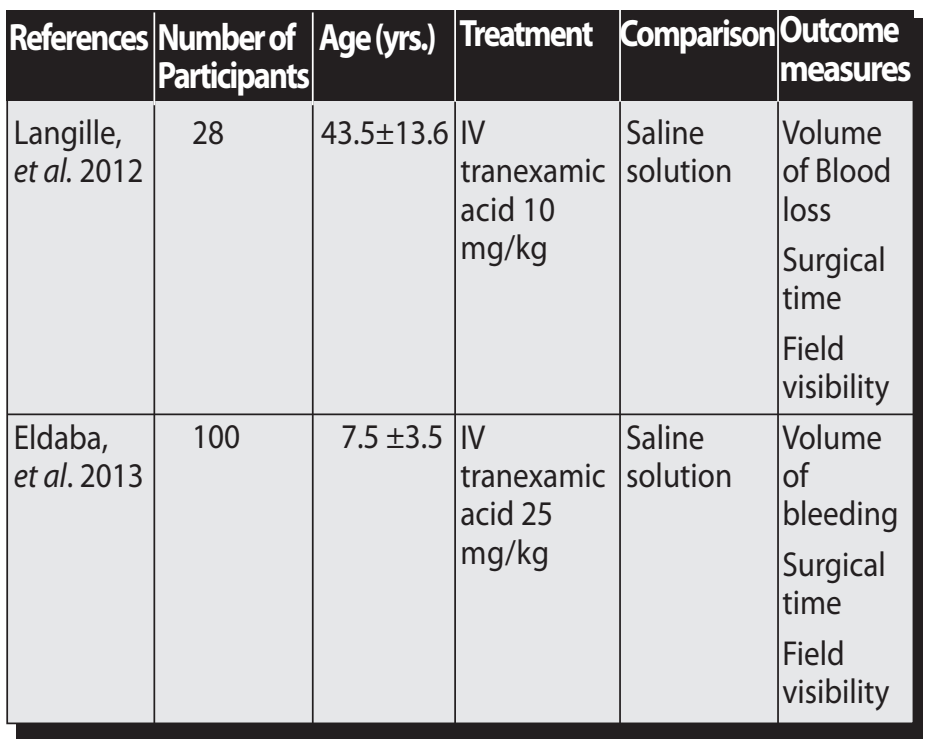

were randomized into 2 groups using a computer based random number generator. The first group was given a slow intravenous injection of tranexamic acid $25 \mathrm{mg} / \mathrm{kg}$ diluted in $10 \mathrm{ml}$ of saline while the second group was given a slow intravenous injection of normal saline. Anesthesiologists, operating personnel and study staff were blinded 
to the treatment groups. The same team performed all procedures. A blinded chief nurse who did not participate in the study protocol prepared the syringes. Outcomes measured in the study were 1) blood loss, which was estimated by weighing sponges and measuring operative suction volume, 2) time of operation, and 3) surgical field

Table 2. Results of Individual Studies

\begin{tabular}{|c|c|c|}
\hline & Langille, et al. & Eldaba, et al. \\
\hline Jadad Score & 4 & 4 \\
\hline $\begin{array}{l}\text { Volume of blood } \\
\text { loss (ml) } \\
\text { Tranexamic acid } \\
\text { Saline }\end{array}$ & $\begin{array}{r}115(30-600) \\
200(100-400)\end{array}$ & $\begin{array}{r}102 \pm 19 \\
153 \pm 3.6\end{array}$ \\
\hline $\begin{array}{l}\text { Surgical Time (minutes) } \\
\text { Tranexamic acid } \\
\text { Saline }\end{array}$ & $\begin{array}{l}121.5(63-152) \\
131.5(83-177)\end{array}$ & $\begin{array}{r}45.2 \pm 12.2 \\
65.5 \pm 13.6 \\
\end{array}$ \\
\hline $\begin{array}{l}\text { Field Visualization } \\
\text { Tranexamic acid } \\
\text { Saline }\end{array}$ & $\begin{array}{l}\text { Wormald Scale Score* } \\
5.8(2.5-8.5) \\
5.8(1-8)\end{array}$ & $\begin{array}{l}\text { Grade 3,Boezaart Scale } \\
\text { (no. of patients) }^{* *} \\
8 \\
24\end{array}$ \\
\hline
\end{tabular}

**Grade 3, Boezaart Scale

*Wormald Scale Score ${ }^{4}$ used in Langille, et al. study

Table 3. Bias Assessment of Included Randomized Controlled Trials: (Jadad score)

\begin{tabular}{|l|c|l|l|c|}
\hline References & Randomization & $\begin{array}{l}\text { Allocation } \\
\text { concealment }\end{array}$ & Blinding & No Drop-outs \\
\hline $\begin{array}{l}\text { Langille, } \\
\text { et al. 2012 }\end{array}$ & Yes & Yes & Yes & Yes \\
\hline $\begin{array}{l}\text { Eldaba, } \\
\text { et al. 2013 }\end{array}$ & Yes & Yes & Yes & Yes \\
\hline
\end{tabular}

visibility, as estimated by the surgeon 15 and 30 minutes after start of surgery using the Boezaart Scale. ${ }^{2}$ Results of the study showed that at the $15^{\text {th }}$ and $30^{\text {th }}$ minute marks, a higher number of participants in group 2 (placebo group) had grade III surgical field or moderate bleeding that lightly compromised the surgical field (respective $p$ values, $p=.06$ and $p=.01$ ). The study also showed a significant difference between the groups for duration of surgery and volume of bleeding, both with $p$ $<.01$. The study showed that intravenous tranexamic acid in children who underwent ESS improved surgical field quality, and reduced intraoperative bleeding and duration of surgery.

Risk of bias is presented in Table 3. All screened patients were randomized, and investigators, surgeons and patients were blinded. There was no evidence of selective drop out, however the data on intention to treat (ITT) was not reported in both studies included.

The forest plot on intraoperative blood loss shows the mean difference of tranexamic acid compared to saline solution in endoscopic sinus surgery. The summary of the observed difference was $-51.00\left(\mathrm{Cl}_{95}\right.$ $[-59.27,-42.73])$ for the $25 \mathrm{mg} / \mathrm{kg}$ tranexamic acid vs. saline in children, and $-85.00\left(\mathrm{Cl}_{95}[-192.95,22.95]\right)$ for $15 \mathrm{mg} / \mathrm{kg}$ tranexamic acid vs. saline in adults. The pooled analysis of the 2 studies had a standardized mean difference of $-51.20\left(\mathrm{Cl}_{95}[-59.44,-42.95]\right)$ showing that the blood loss in $\mathrm{ml}$ was lower in the tranexamic acid group compared to saline solution. (Figure 2)

The forest plot of surgical time shows the mean difference of tranexamic acid compared to saline solution in endoscopic sinus surgery. The summary of the observed difference was $-20.00\left(\mathrm{Cl}_{95}\right.$ $[-25.06,-14.94])$ for the $25 \mathrm{~m} / \mathrm{kg}$ tranexamic acid vs. saline in children, and $-10.00\left(\mathrm{Cl}_{95}[-28.06,8.72]\right)$ for $15 \mathrm{mg} / \mathrm{kg}$ tranexamic acid vs. saline in adults. The pooled analysis of the 2 studies had a standardized mean

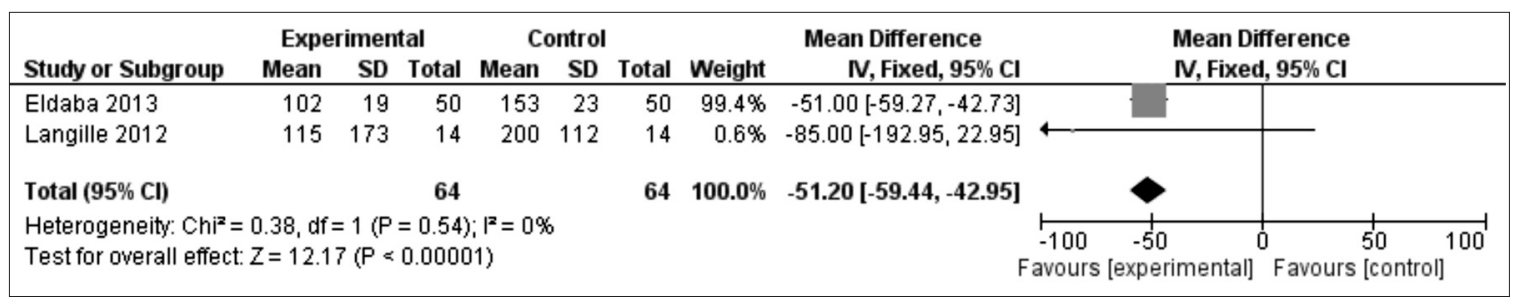

Figure 2. Forest Plot of Primary Endpoint comparing I.V. tranexamic acid vs saline solution on Intraoperative Blood Loss

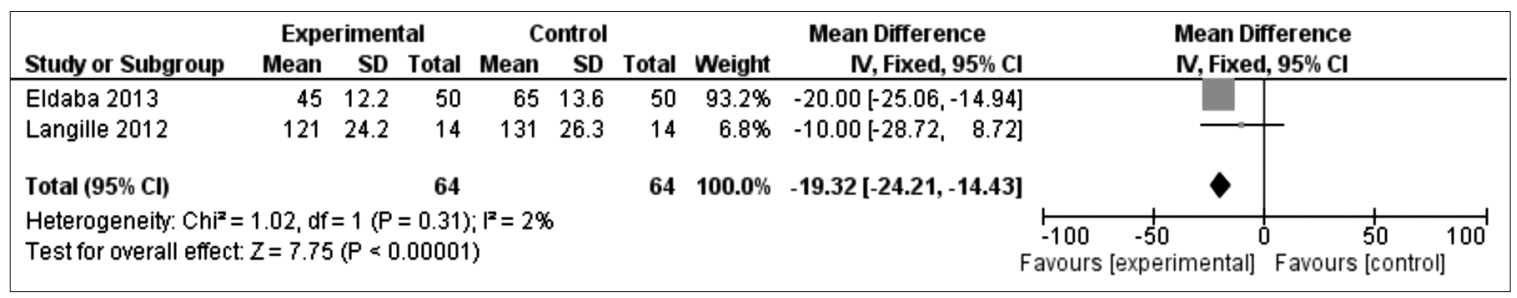

Figure 3. Forest Plot of Primary Endpoint comparing I.V. tranexamic acid vs saline solution on Surgical Time 
difference of $-19.32\left(\mathrm{Cl}_{95}[-24.21,-14.43]\right)$ showing that the surgical time in minutes was shorter in the tranexamic group compared to saline solution. (Figure 3)

The secondary outcome on surgical field visualization was not pooled together because the 2 studies used different scales of evaluation.

\section{DISCUSSION}

This systematic review showed that intravenous tranexamic acid reduced blood loss and surgical time in patients who underwent endoscopic sinus surgery compared to saline solution. The overall treatment effect was $51.2 \%$ less blood loss and $19.32 \%$ less surgical time. In the practice of otolaryngology, endoscopic sinus surgery is one of the common nasal surgeries and bleeding is a big concern. Bleeding can cause serious complications due to interference with visibility and quality of the surgical field. Poor visibility can cause greater blood loss that may lead to morbidities and possible mortality. Tranexamic acid is a hydrophilic antifibrinolytic agent that can be given to reduce bleeding. Evidence that tranexamic acid reduces bleeding in ESS has been available for many years. It has been used in different surgeries to reduce perioperative bleeding with successful results. ${ }^{6}$

This systematic review pooled analysis of 2 very good quality randomized controlled trials with methodological assessments of 4 (Jadad Score). The 2 main primary outcomes of blood loss and surgical time had the same methods of measurement, recorded in milliliters and minutes, respectively. The observation periods for the 2 studies were consistent (during, and before the end of the operation). The risk of follow-up bias was not a problem in the 2 studies because all observations were made perioperatively.

This systematic review had several limitations. First, the age groups were different-- the Langille study ${ }^{5}$ had adult patients and the Eldaba study $^{2}$ had children as patients. The doses of tranexamic acid given intravenously were different-- $15 \mathrm{mg} / \mathrm{kg}$ for the Langille study and 25 $\mathrm{mg} / \mathrm{kg}$ for the Eldaba group. The scales used for field visualization were different-- Langille used the Wormald grading scale ${ }^{9}$ and Eldaba used the Boezaart scoring scale. ${ }^{10}$ Therefore, there no concrete evidence on the quality of field visualization that can be pooled.

In spite of these limitations, the pooled analysis was favorable for I.V. tranexamic acid in reduction of blood loss and surgical time. Cumulative data from the included studies showed that there was a significant improvement in field visibility and decrease in blood loss that could further lead to reduced operation time. This finding can be beneficial to otorhinolaryngologists in preoperative planning of their sinus cases. However, the study is limited by the trials included, showing that there is a lack of data on the subject. Limited data retrieval shows that there is room for more studies to be done on this subject. The authors recommend more randomized control trials to further strengthen the significance of tranexamic acid in ESS or resolve any uncertainties regarding its use.

In conclusion, intravenous tranexamic acid reduces blood loss and shortens surgical time in endoscopic sinus surgery among patients with chronic rhinosinusitis. However, the additional benefit of tranexamic acid for better field visualization is not clear. Adverse effects were not considered in this study but intraoperative tranexamic acid is an option for endoscopic sinus surgeries with blood loss as a concern. Further randomized clinical trials are needed to strengthen the evidence on the effectivity of tranexamic acid for endoscopic sinus surgeries.

\section{REFERENCES}

1. Khafagy A, Osman S. Does tranexamic acid, deliberate hypotension, and anti-Trendelenburg position improve the quality and outcome for functional endoscopic sinus surgery. Egypt J Otolaryngol. 2013;29:71-75. [cited 2014 Aug 12]; Available at: http://www.ejo.eg.net/text. asp?2013/29/2/71/134334 DOI: 10.7123/01.EJO.0000426359.69695.7c.

2. Eldaba $A A, A m r Y M$, Albirmawy OA. Effects of tranexamic acid during endoscopic sinus surgery in children. Saudi J Anesth. 2013 Jul; 7(3):229-233. DOI: 10.4103/1658-354X.115314. PMID: 24015121 PMCID: PMC3757791.

3. Fromme GA, MacKenzie RA, Gould AB Jr, Lund BA, Offord KP. Controlled hypotension for orthognathic surgery. Anesth Analg. 1986 Jun; 65(6):683-6. PMID: 3706806.

4. Kim RJT, Douglas RG. Perioperative care for functional endoscopic sinus surgery. The Otorhinolaryngologist. 2012; 5(1):27-30. [cited 2014 Aug 12]. Available at: http://www. greenlaneresearch.co.nz/Portals/1/Docs/The\%200torhinolaryngologist\%202012\%20Kim.pdf.

5. Langille MA, Chiarella A, Cote DW, Mulholland G, Sowerby LJ, Dziegielewski PT, Wright ED. Intravenous tranexamic acid and intraoperative visualization during functional endoscopic sinus surgery: a double-blind randomized control trial. Int Forum Allergy Rhinol. 2013 Apr; 3(4): 315-8. DOI: 10.1002/alr.21100. PMID: 23044919.

6. Ker K, Edwards P, Perel P, Shakur H, Roberts I. Effect of tranexamic acid on surgical bleeding: systematic review and cumulative meta-analysis. BMJ. 2012 May 17; 344: e3054. DOI: 10.1136/ bmj.e3054. PMID: 22611164. PMCID: PMC3356857.

7. Abbasi H, Behdad S, Ayatollahi V, Nazemian N, Mirshamsi P. Comparison of two doses of tranexamic acid on bleeding and surgery site quality during sinus endoscopy surgery. Adv Clin Exp Med. 2012 Nov-Dec; 21(6):773-780. PMID: 23457130

8. Thongrong C, Kasemsiri P, Carrau RL, Bergese SD. Control of bleeding in endoscopic skull base surgery: current concepts to improve hemostasis. ISRN Surg. 2013 Jun 13; 2013: 191543. DOI: 10.1155/2013/191543. PMID: 23844295 PMCID: PMC3697291.

9. Athanasiadis T, Beule A, Embate J, Steinmeier E, Field J, Wormald PJ. Standardized videoendoscopy and surgical field grading scale for endoscopic sinus surgery: a multi-centre study. Laryngoscope. 2008 Feb; 118(2):314-319. DOI: 10.1097/MLG.0b013e318157f764. PMID: 17989575.

10. Boezaart AP, van der Merwe J, Coetzee A. Comparison of sodium nitroprusside and esmololinduced controlled hypotension for functional endoscopic sinus surgery. Can J Anaesth. 1995 May; 42(5 Pt 1):373-6. DOI: 10.1007/BF03015479. PMID: 7614641.

11. Jadad Scale for Reporting Randomized controlled trials. In: Halpern SH, Douglas MJ, editors. Evidence-based obstetric anesthesia. Oxford: Blackwell Publishing Ltd.; 2005. p. 237-238. DOI: 10.1002/9780470988343.app1

12. Jadad AR, Moore RA, Carroll D, Jenkinson C, Reynolds DJ, Gavaghan DJ, et al. Assessing the quality of reports of randomized clinical trials: is blinding necessary? Control Clin Trials 1996 Feb; 17(1):1ñ12. PMID: 8721797

13. Wright ED, Agrawal S. Impact of perioperative systemic steroids on surgical outcomes in patients with chronic rhinosinusitis with polyposis: evaluation with the novel perioperative sinus endoscopy (POSE) scoring system. Laryngoscope. 2007 Nov; 117(11 Pt 2 Suppl 115):1-28 DOI: 10.1097/MLG.0b013e31814842f8. PMID: 18075447.

14. Fawzy M, Ewieda T. Tranexamic acid versus hypotensive anesthesia using isoflurane for improvement of outcome of functional endoscopic sinus surgery. PAN Arab Journal of Rhinology. 2014 Mar; 4(1): 32-39. 